\title{
Associações demográficas e clínicas com pneumonia hospitalar adquirida e associada à ventilação mecânica
}

\author{
Karla Karoline Bacellar ${ }^{1}$, Rejane Kiyomi Furuya², Jucinay Phaedra Silva Sanches ${ }^{3}$, Gilselena \\ Kerbauy ${ }^{4}$, Renata Aparecida Belei5 ${ }^{5}$ Flávia Meneguetti Pieri6
}

\section{RESUMO}

Objetivo: verificar a associação de características sociodemográficas e clínicas com Pneumonia Associada à Ventilação Mecânica e Pneumonia Hospitalar Adquirida. Método: estudo transversal com dados secundários, obtidos das fichas de notificação das infecções relacionadas à assistência à saúde ocorridas entre janeiro de 2017 e dezembro de 2018, cujas variáveis foram analisadas por estatística descritiva e inferencial. Resultados: a Pneumonia Associada à Ventilação Mecânica foi mais frequente em indivíduos entre 18 e 59 anos e a Pneumonia Hospitalar Adquirida em indivíduos com 60 anos ou mais $(p=0,003)$. Quanto à unidade onde a pneumonia foi diagnosticada, houve maior frequência de Pneumonia Associada à Ventilação Mecânica em Unidade de Terapia Intensiva, Centro de Tratamento de Queimados e Unidade de Terapia Intensiva de Queimados ( $p<0,001$ ). As frequências de óbito foram 48,9\% nos casos com Pneumonia Hospitalar Adquirida e 69,2\% nos casos de Pneumonia Associada à Ventilação Mecânica $(p<0,001)$. Houve associação significante entre cultura bacteriana e o tipo de pneumonia, sendo que, no grupo Pneumonia Associada à Ventilação Mecânica , 76,0\% dos pacientes tiverem cultura positiva, comparado a $56,3 \%$ no grupo Pneumonia Hospitalar Adquirida $(p<0,001)$. Os microrganismos mais frequentes foram Acinetobacter baumannii, Klebsiella pneumoniae, Staphylococcus aureus e Pseudomonas aeruginosa. Conclusão: houve associação estatisticamente significante entre o tipo de pneumonia e idade, sendo a Pneumonia Associada à Ventilação Mecânica mais frequente em jovens, e a Pneumonia Hospitalar Adquirida , em idosos. Também houve associação significante entre o tipo de pneumonia e a unidade, sendo mais frequente a Pneumonia Associada à Ventilação Mecânica nas Unidades de Terapia Intensiva e Queimados. O óbito foi mais frequente nos casos de Pneumonia Associada à Ventilação Mecânica , com associação significante.

Descritores: Pneumonia Associada à Ventilação Mecânica; Pneumonia Associada a Assistência à Saúde; Infecção Hospitalar; Enfermagem; Fatores de Risco.

1 Enfermeira. Bacharel em Enfermagem. Universidade Estadual de Londrina. Londrina, Paraná, Brasil. karla.bacellar@live.com. ORCID: 0000-0002-3067-4175

2 Enfermeira. Doutora em Ciências pela Escola de Enfermagem de Ribeirão Preto da Universidade de São Paulo. Professora do Instituto Federal do Paraná - Campus Londrina. Londrina, Paraná, Brasil. rejane.furuya@ifpr.edu.br. ORCID: 0000-0003-0885-5364

3 Enfermeira. Mestre em Enfermagem pela Universidade Estadual de Londrina. Enfermeira da Comissão de Controle de Infeç̧ão Hospitalar da Irmandade da Santa Casa de Londrina. Londrina, Paraná, Brasil. jucinayps2@gmail.com. ORCID: 0000-0002-04727659

4 Enfermeira. Doutora em Microbiologia pela Universidade Estadual de Londrina. Professor Adjunto do Departamento de Enfermagem da Universidade Estadual de Londrina. Londrina, Paraná, Brasil. gilselena@hotmail.com. 0000-0002-1737-4282

5 Enfermeira. Doutora em Educação pela Universidade Estadual Paulista Júlio de Mesquita Filho. Enfermeira da Comissão de Controle de Infecção Hospitalar do Hospital Universitário da Universidade Estadual de Londrina. Londrina, Paraná, Brasil. rabelei@yahoo.com.br. ORCID: 0000-0003-2386-6360

6 Enfermeira. Doutora em Ciências pela Escola de Enfermagem de Ribeirão Preto da Universidade de São Paulo. Professora Adjunta do Departamento de Enfermagem da Universidade Estadual de Londrina. Londrina, Paraná, Brasil. fpieri@uel.br. ORCID: 0000-0003$1239-2550$

\section{Autor Correspondente}

Flávia Meneguetti Pieri

Endereço: Universidade Estadual de Londrina, Centro de Ciências da

Saúde. Avenida Robert Koch, 60. Vila Operária. CEP: 86038-350.

Londrina, Paraná, Brasil.

Telefone: (43) 33712249. Fax: (43) 33712249

E-mail: fpieri@uel.br

Data de submissão: 02/04/2020

Data de aceite: $21 / 07 / 2020$

\section{Como citar esse artigo:}

BACELLAR, K.K. et al. Associações demográficas e clínicas com pneumonia hospitalar adquirida e associada à ventilação mecânica. Advances in Nursing and Health, v. 2, p. 17-33, Londrina, 2020. 


\section{INTRODUÇÃO}

As Medidas de Prevenção de Infecção Relacionada à Assistência à Saúde (IRAS) foram revisadas em 2017 pela Agência Nacional de Vigilância Sanitária (ANVISA), com o intuito de manter atualizados os protocolos de medidas de prevenção para a redução das IRAS no cenário brasileiro(1). Em 2019 os critérios diagnósticos das infecções sofreram alterações, incluindo a Pneumonia Associada à Ventilação Mecânica (PAV) e a Pneumonia Hospitalar Adquirida (PHA)(2).

Considera-se como PAV as infecções por um período maior que dois dias de calendário, sendo que o Dia 1 (D1) é o dia de início da ventilação mecânica (VM) e, na data da infecção, o paciente estava em VM ou o respirador mecânico havia sido removido no dia anterior(2-3).

As taxas de PAV podem variar conforme a população de pacientes afetada. De acordo com o estudo de Manfredini et al.(4), a incidência aumenta com a permanência da VM e apontam taxas de aproximadamente $3 \%$ por dia durante os primeiros cinco dias de VM e, depois, $2 \%$ para cada dia. No que tange à mortalidade, 33\% dos hospitalizados com PAV evoluíram para óbito com associação direta à infecção.

Entre as principais ocorrências de IRAS, verifica-se a PHA, que é adquirida no serviço de saúde, mas não associada ao uso da $\mathrm{VM}^{(2)}$. No momento, como praticamente não existem requisitos para monitorar ou relatar os casos de PHA, é menos provável que os hospitais monitorem sua própria incidência e os danos $\operatorname{associados}^{(5)}$.

No estudo de Luyt et al.(6), a prevenção de PHA e PAV continua sendo uma tarefa difícil e complexa. Houve uma relação entre os principais microrganismos responsáveis, áreas geográficas, condições clínicas e demográficas do paciente em Unidade de Terapia Intensiva (UTI), tempo de hospitalização, permanência na UTI, entre outros fatores de risco.

Em estudo realizado em 183 hospitais com 11.282 pacientes, dos quais 452 tiveram uma ou mais IRAS, pesquisadores revisaram, retrospectivamente, registros médicos e estimaram que havia 648.000 pacientes com 721.800 IRAS em unidades hospitalares dos EUA em 2011(7).

Em 2020 Patty et al.(8) demonstraram a incidência de PHA foi de 0,64 casos por 
1.000 pacientes/dia. Os fatores associados com maior destaque foram a idade avançada e a presença de comorbidades. Como fator protetor ao desenvolvimento desta infecção, houve destaque para a elevação da cabeceira do leito, com redução em $26 \%$ das chances de desenvolver a infecção.

Ressalta-se que as IRAS atingem em média $14 \%$ das internações em nível nacional e dos 234 milhões de pacientes que são operados por ano no mundo, em média, um milhão morre em decorrência $\operatorname{destas}^{(9)}$.

Para Souza et al.(10), o alto risco de mortalidade relacionada às IRAS está intimamente associado à realização de procedimentos invasivos diagnósticos e terapêuticos, à gravidade da doença de base que acomete 0 paciente, ao sítio da infecção, à adequação da terapia e à sensibilidade dos microrganismos aos antimicrobianos.

Atualmente, existem diversas estratégias de prevenção baseados em evidências científicas que são comprovadamente eficazes quando realizada

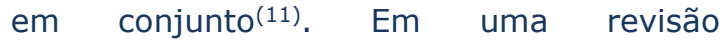
integrativa, foram analisados diversos estudos sobre estratégias de prevenção das
PAV. Desses, 95,6\% indicaram a elevação da cabeceira entre $30^{\circ}$ e $45^{\circ} ; 82,6 \%$ indicaram higiene oral; e $60,8 \%$ indicaram o despertar diário de desmame de sedação. A profilaxia para úlcera gástrica foi mencionada em $47,8 \%$ dos artigos. Em frequência mais baixa, manutenção do manguito foi relatada em 43,4\%, e aspiração subglótica, em $21,7 \%$.

Um estudo observacional, realizado em 21 hospitais de um sistema de saúde integrado, realizou intervenções que incluíram cuidados básicos da enfermagem, a fim de prevenir a PHA, os quais incluíram mobilização, alimentação vertical, avaliação da deglutição, restrições de sedação, cabeceira elevada e cuidados bucais. Tais medidas resultaram em uma redução significativa das PHA de 5,92 para 1,79 por 1000 admissões(12).

Nessa perspectiva, foi criado um pacote de medidas para prevenção de PAV, atualmente denominado bundles, proposto pelo Centro de Controle e Prevenção de Doenças dos Estados Unidos (CDC) (2003)(13), baseadas em evidências científicas elencadas na campanha 5 milhões de vidas do Institute for Healthcare Improvement $(\mathrm{IHI})^{(14)}$. Considerando a 
gravidade da doença e com base nos fatores de risco para as pneumonias relacionadas à assistência à saúde, ANVISA (2017)(1) listou as principais medidas para a prevenção divididas entre as medidas gerais, medidas específicas e outras medidas para a vigilância de cada uma das pneumonias no ambiente hospitalar.

$$
\text { O Ministério da Saúde }
$$
implantou, por meio da Portaria no 2.216 de 1998, a Comissão de Controle de Infecções Hospitalares (CCIH), que trabalha na redução da incidência e gravidade na evolução dos pacientes internados, redução do custo hospitalar por meio do controle e prevenção de IRAS aplicando os bundles. Vale destacar que as ações da enfermagem são essenciais para o desenvolvimento dos serviços, pois, além de fiscalizar rotineiramente as unidades e o desenvolvimento do trabalho dos profissionais da saúde, elabora e atualiza os procedimentos operacionais padrão, realiza vigilância epidemiológica das IRAS, fortalece o conhecimento dos profissionais por meio da educação permanente e continuada, dentre outras funções(14).

Com base nos argumentos aqui citados, o objetivo deste estudo foi verificar a associação de características sociodemográficas e clínicas com PAV e PHA.

\section{MÉTODO}

Trata-se de um estudo analítico, transversal e quantitativo, que utilizou como fonte de informação dados secundários obtidos de fichas de notificação das IRAS. O estudo foi realizado no Hospital Universitário da Universidade Estadual de Londrina (HU/UEL), localizado na cidade de Londrina, Paraná, Brasil.

A população estudada abrangeu todos os casos notificados de indivíduos que apresentaram PHA ou PAV. Os critérios de inclusão foram pacientes adultos, com idade a partir de 18 anos, internados no ProntoSocorro (PS), Unidade de Internação (UI), UTI, Centro de Tratamento de Queimados (CTQ) ou Unidade de Terapia Intensiva de Queimados (UTQ) e diagnosticados com PHA ou PAV, no período de janeiro de 2017 a dezembro de 2018. Os diagnósticos de PHA e PAV foram realizados pela médica da CCIH, conforme a classificação Código Internacional de Doenças (CID-10) e definição da ANVISA(3). O critério de exclusão foram casos notificados em que as fichas estavam com os dados incompletos. 
A identificação de pacientes foi realizada por busca ativa, considerando-se pacientes com prescrição de antimicrobiano(s). No hospital onde o estudo foi realizado, a antibioticoterapia é iniciada somente após a liberação da CCIH. Desta forma, com o trabalho desta comissão, é possível identificar todos os pacientes que tiveram antimicrobianos prescritos. Após a identificação dos pacientes com algum tipo de infecção, é aberta uma ficha de suspeita de IRAS; e, para os casos confirmados, passa-se a utilizar a ficha de notificação das IRAS.

A ficha de notificação de IRAS foi elaborada pela equipe da CCIH de acordo com os critérios estabelecidos pela ANVISA(3), contendo os itens clínicos, laboratoriais e de imagem. O preenchimento é realizado por voluntários da graduação/estagiários da $\mathrm{CCIH}$, treinados pela enfermeira, doutora em educação, responsável pelo setor, no decorrer da internação do paciente, ou seja, os estagiários fazem o acompanhamento e atualizam as informações, desde a notificação até a alta ou óbito.

Foram coletados todos os dados da ficha de notificação de IRAS, por meio de digitação em planilha Excel. Vale ressaltar que esta ficha já estava em uso antes do período de coleta de dados e se mostrou completa para a equipe de CCIH quanto aos dados relacionados às IRAS.

A etapa de coleta de dados foi realizada no período de março a dezembro de 2019, por dois pesquisadores treinados pela pesquisadora orientadora desta pesquisa. A coleta de dados foi realizada na sala da $\mathrm{CCIH}$, uma vez por semana, no período vespertino.

Foram coletadas seguintes variáveis demográficas e clínicas: sexo (masculino ou feminino), idade (em anos), dias de internação, dias de internação até o diagnóstico de pneumonia, unidade de admissão e unidade onde a pneumonia foi diagnosticada (PS, UI, UTI, CTQ ou UTQ), tipo de pneumonia (PHA ou PAV), culturas diagnósticas (aspirado de secreção traqueal e/ou hemocultura), tipo de microrganismo e óbito.

As culturas diagnósticas foram realizadas conforme a solicitação do médico responsável pelo paciente, considerando-se aspectos clínicos (hipertermia, mudança nas características da secreção traqueal), laboratoriais (resultados de leucograma) e de 
imagem (raio X).

A coleta de secreção traqueal foi realizada por meio de aspiração das vias aéreas, com uso de cateter. A hemocultura foi realizada a partir de duas punções de veia ou artéria periférica e coleta de sangue em frascos de hemocultura aeróbico e anaeróbico. Após a coleta o material, foi encaminhado ao laboratório em análises clínicas do HU/UEL, para análise incubatória da secreção traqueal e da hemocultura pelo sistema automatizado Bactec ${ }^{\mathrm{TM}}$.

As variáveis numéricas foram categorizadas da seguinte forma: idade (18 a 59 anos e 60 ou mais), dias de internação (até 30 dias e 31 ou mais) e dias de internação até o diagnóstico (até 14 dias e 15 ou mais).

A análise de dados foi realizada no programa Statistical Package for the Social Sciences (SPSS ${ }^{\circledR}$ ), versão 20.0. A PHA e a PAV foram as variáveis independentes associadas com as variáveis dependentes: sexo, idade, tempo de internação, tempo de internação até o diagnóstico, unidade de internação, óbitos e culturas diagnósticas, por meio do Teste Qui-Quadrado. O nível de significância adotado foi de 5\%.

O estudo foi aprovado pelo Comitê de
Ética em Pesquisa da Universidade Estadual de Londrina (CEP/UEL), de acordo com as Diretrizes e Normas Regulamentadoras de Pesquisa em Seres Humanos, Resolução no 466/2012 do Conselho Nacional de Saúde (CNS), sob Certificado de Apresentação para Apreciação Ética (CAAE) n 00745218.0.0000.5231, Parecer $n^{\circ}$ 2.978.943, emitido em 24 de outubro de 2018.

\section{RESULTADOS}

$$
\text { No período analisado, foram }
$$
notificados 573 casos de PHA e 146 de PAV, totalizando 719 casos, sendo que todos os casos notificados constituíram a amostra deste estudo. Dentre os 719 casos notificados, dois pacientes tiveram tanto a notificação de PHA como de PAV.

Considerando-se ambos os tipos de pneumonia, a maioria era do sexo masculino. Com relação à idade, houve maior frequência de idosos com PHA, enquanto que, para PAV, os indivíduos até 59 anos foram mais frequentes. A associação entre a idade e o tipo de pneumonia foi significante $(p=0,003)$ (Tabela 1). 
Tabela 1 - Análise descritiva das características demográficas e clínicas dos 719 casos de pneumonia conforme o tipo de pneumonia e valores de probabilidade (p) associados ao Teste Qui-Quadrado. Londrina, PR, Brasil, 2019

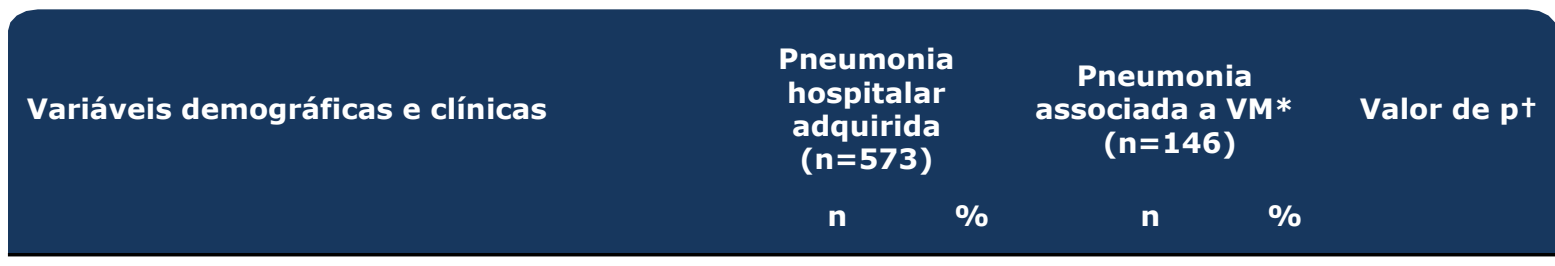

Sexo

\begin{tabular}{llllll} 
Masculino & 355 & 62,0 & 89 & 61,0 & 0,825 \\
Feminino & 218 & 38,0 & 57 & 39,0 & \\
\hline
\end{tabular}

Idade(anos)

18 a 59

$227 \quad 39,6$

$78 \quad 53,4$

60 ou mais

$346 \quad 60,4$

$68 \quad 46,6$

0,003

Dias de internação

Até $\mathbf{3 0}$

$379 \quad 66,1 \quad 98 \quad 67,1$

31 ou mais

$194 \quad 33,9$

$48 \quad 32,9$

0,823

Dias de internação até o diagnóstico de pneumonia

$\begin{array}{lrrrrr}\text { Até } 14 \text { dias } & 480 & 83,8 & 126 & 86,3 & 0,453 \\ \mathbf{1 5} \text { ou mais } & 93 & 16,2 & 20 & 13,7 & \end{array}$

Unidade de admissão

$\begin{array}{lrrrrr}\text { PS }^{\ddagger} & 451 & 78,8 & 101 & 69,2 & \\ \text { UIฐ } & 61 & 10,6 & 5 & 3,4 & <0,001 \\ \text { UTI/UTQ/CTQ\$ } & 61 & 10,6 & 40 & 27,4\end{array}$

\begin{tabular}{|c|c|c|c|c|c|}
\hline \multicolumn{6}{|c|}{$\begin{array}{l}\text { Unidade onde a pneumonia foi } \\
\text { diagnosticada }\end{array}$} \\
\hline $\mathbf{P S}^{+}$ & 222 & 38,7 & 38 & 26,1 & \\
\hline $\mathbf{U I}^{\ddagger}$ & 166 & 29,0 & 10 & 6,8 & $<0,001$ \\
\hline UTI/UTQ/CTQII & 185 & 32,3 & 98 & 67,1 & \\
\hline \multicolumn{6}{|l|}{ Óbito } \\
\hline Sim & 280 & 48,9 & 101 & 69,2 & \\
\hline Não & 293 & 51,1 & 45 & 30,8 & $<0,001$ \\
\hline
\end{tabular}

*Ventilação Mecânica; †Teste Qui-Quadrado; `Pronto-Socorro; §Unidade de Internação; IIUnidade de Terapia Intensiva, Unidade de Terapia Intensiva de Queimados e Centro de Tratamento de Queimados. 
Com relação ao tempo de internação, em ambos os grupos, o período até 30 dias foi mais frequente e, no que se refere aos dias de internação até o diagnóstico de pneumonia, se destacou até 14 dias. Não houve associação significante entre o tipo de pneumonia e o tempo de internação total e para o diagnóstico da doença.

A unidade de admissão com maior número de casos foi o PS. Houve associação significante entre a unidade de admissão e o tipo de pneumonia $(p<0,001)$, com maior frequência de PAV no grupo UTI/UTQ/CTQ. Também houve associação significante entre a unidade onde a pneumonia foi diagnosticada e o tipo de pneumonia $(p<0,001)$, com maior frequência de PAV no grupo UTI/UTQ/CTQ.

Em 48,9\% dos casos notificados de PHA e $69,2 \%$ dos casos de PAV, o paciente evoluiu a óbito, com associação significante entre óbito e o tipo de pneumonia $(p<0,001)$.

Em 574 casos $(79,8 \%)$, foram realizadas culturas diagnósticas (aspirado de secreção traqueal e/ou hemocultura). Em ambos os grupos, a maioria dos casos teve cultura positiva $(\mathrm{PHA}=56,3 \% \quad \mathrm{e}$ PAV $=76,0 \%$, sendo significante a associação entre cultura positiva e tipo de pneumonia $(p<0,001)$.

Com relação ao tipo de microrganismo, os mais frequentes foram Acinetobacterbaumannii,

Klebsiellapneumoniae, Staphylococcus aureus e Pseudomonasaeruginosa. A associação entre o microrganismo e o tipo de pneumonia foi significante apenas para Staphylococcus aureus $(p<0,001)$, sendo que a presença do microrganismo foi mais frequente nos casos de PAV, quando comparados com a PHA (Tabela 2).

\section{DISCUSSÃO}

Propôs-se, com este estudo, descrever as características demográficas e clínicas dos casos de pneumonia e associar essas variáveis com o tipo de pneumonia (PHA ou PAV).

Os resultados deste estudo mostram no perfil da amostra a prevalência do sexo masculino e a faixa etária acima de 60 anos para os casos de PHA e até 59 anos para os casos de PAV. O estudo de Baker e Quinn(15) confirma que pacientes mais jovens também correm algum risco e adquirem pneumonia enquanto estão hospitalizados. 
Tabela 2 - Análise descritiva das culturas diagnósticas dos 574 casos de pneumonia conforme o tipo de pneumonia e valores de probabilidade ( $p$ ) associados ao Teste QuiQuadrado. Londrina, PR, Brasil, 2019

Variáveis

Pneumonia

hospitalar

adquirida

$(n=428)$

n
Pneumonia

associada a

VM*

$(n=146)$

$\%$
Valor de

$\%$

\section{Cultura diagnóstica positiva}

\begin{tabular}{|c|c|c|c|c|c|}
\hline Sim & 241 & 56,3 & 111 & 76,0 & \multirow[t]{2}{*}{$<0,001$} \\
\hline Não & 187 & 43,7 & 35 & 24,0 & \\
\hline \multicolumn{6}{|c|}{ Acinetobacterbaumannii } \\
\hline $\begin{array}{l}\text { Sim } \\
\text { Não }\end{array}$ & $\begin{array}{l}109 \\
319\end{array}$ & $\begin{array}{l}25,5 \\
74,5\end{array}$ & $\begin{array}{r}39 \\
107\end{array}$ & $\begin{array}{l}26,7 \\
73,3\end{array}$ & 0,766 \\
\hline \multicolumn{6}{|c|}{ Klebsiellapneumoniae } \\
\hline Sim & 63 & 14,7 & 24 & 16,4 & \multirow[b]{2}{*}{0,617} \\
\hline Não & 365 & 85,3 & 122 & 83,6 & \\
\hline \multicolumn{6}{|c|}{ Staphylococcus aureus } \\
\hline Sim & 52 & 12,1 & 34 & 23,3 & \multirow[b]{2}{*}{$<0,001$} \\
\hline Não & 376 & 87,9 & 112 & 76,7 & \\
\hline \multicolumn{6}{|c|}{ Pseudomonasaeruginosa } \\
\hline Sim & 50 & 11,7 & 17 & 11,6 & \multirow[b]{2}{*}{0,990} \\
\hline Não & 378 & 88,3 & 129 & 88,4 & \\
\hline \multicolumn{6}{|c|}{ Cândida spp } \\
\hline Sim & 42 & 9,8 & 12 & 8,2 & \multirow[t]{2}{*}{0,569} \\
\hline Não & 386 & 90,2 & 134 & 91,8 & \\
\hline \multicolumn{6}{|c|}{ Estafilococos coagulase negativa } \\
\hline Sim & 25 & 5,8 & 11 & 7,5 & \multirow[b]{2}{*}{0,466} \\
\hline Não & 403 & 94,2 & 135 & 92,5 & \\
\hline \multicolumn{6}{|c|}{ Cocos Gram-positivos } \\
\hline Sim & 21 & 4,9 & 4 & 2,7 & \multirow{2}{*}{0,268} \\
\hline Não & 407 & 95,1 & 142 & 97,3 & \\
\hline \multicolumn{6}{|c|}{ Enterobacterclocae } \\
\hline Sim & 14 & 3,3 & 8 & 5,5 & \multirow[b]{2}{*}{0,230} \\
\hline Não & 414 & 96,7 & 138 & 94,5 & \\
\hline \multicolumn{6}{|c|}{ Escherichia coli } \\
\hline Sim & 14 & 3,3 & 5 & 3,4 & \multirow[b]{2}{*}{0,929} \\
\hline Não & 414 & 96,7 & 141 & 96,6 & \\
\hline \multicolumn{6}{|c|}{ Serratiamarcescens } \\
\hline Sim & 11 & 2,6 & 8 & 5,5 & \multirow[b]{2}{*}{0,900} \\
\hline Não & 417 & 97,4 & 138 & 94,5 & \\
\hline
\end{tabular}

*Ventilação Mecânica; 'Teste Qui-Quadrado. 
Devido ao alto índice de comorbidades enfrentadas pela população idosa, no Brasil, essa faixa etária possui alta prevalência de hospitalizações(16). O hospital que foi cenário da pesquisa também sofre com essa realidade. Além de ser referência para pacientes graves, atende alto número de idosos com doenças crônicas e suas complicações fisiológicas.

Uma justificativa é de que os mesmos, ao longo da vida, desenvolvem comorbidades que fazem com que fiquem mais suscetíveis a doenças, possuindo diminuição em suas atividades, qualidade de vida, consequentemente, elevando a frequência de internação(10). No estudo que avaliou os fatores de risco para PHA, foi descrito que pacientes em todos os tipos de unidades hospitalares correm algum risco para desenvolver PHA; destaca, ainda, risco aumentado para aqueles submetidos à cirurgia(15)

Houve associação significante entre a idade e o tipo de pneumonia; nos pacientes com 18 a 59 anos, a PAV foi mais frequente. Isso se justifica pelo fato de jovens serem mais entubados do que idosos, visto que, muitas vezes, nos serviços hospitalares, incluindo a instituição do presente estudo, pacientes com prognóstico desfavorável e idade avançada são tratados com medidas não invasivas, com a aceitação dos familiares do paciente. Essas medidas que visam propiciar o conforto, aliviar sintomas que trazem sofrimento ao paciente, além de reconhecer a morte como processo natural, se caracterizam por cuidados paliativos(16-18). Em nosso estudo, a maioria dos pacientes foi diagnosticada com pneumonia em até 14 dias de hospitalização. Em estudo sobre PAV em UTI, houve associação positiva entre a ocorrência de pneumonia e o tempo de internação $>15$ dias e o tempo de VM >10 dias(19). Em contrapartida, há uma forte discussão pelos pesquisadores em defesa de que a UTI, apesar de apresentar maior risco para o desenvolvimento da pneumonia, atua de forma sistematizada cumprindo os protocolos de prevenção à $\operatorname{PAV}^{(11,17-18)}$.

Com relação à unidade onde a pneumonia foi diagnosticada, mais de um terço dos casos ocorreram no PS. Apesar de ser considerada uma unidade de porta de entrada, no serviço analisado, os pacientes permanecem hospitalizados, sendo, frequentemente, entubados. De acordo com o estudo de Di Pasquale et al.(20), setenta e três por cento dos casos de PHA tiveram 


\section{t}

origem fora da UTI.

Corroborando ainda esta discussão, um estudo realizado no serviço de urgência e emergência enfatizou alguns fatores que contribuem para este cenário, sendo eles: a demanda espontânea, a superlotação, a longa permanência de pacientes nestas unidades e $\quad$ a falta de materiais/leitos/recursos humanos. Torna-se necessária uma reorganização dos processos de trabalho em prol da redução desses fatores de risco modificáveis, para o desenvolvimento de $\mathrm{PHA}^{(21)}$.

Concomitante a isso, é importante salientar que o perfil dos pacientes atendidos nos PS varia de baixa complexidade até os mais graves. Em estudo exploratório realizado no PS, na cidade de Boa Vista, Roraima, em um hospital semelhante ao do nosso estudo, por se tratar de instituição vinculada ao ensino e de alta complexidade, apontou que $69,28 \%$ dos pacientes foram classificados como de cuidados intensivos; $13,86 \%$ em cuidados semi-intensivos; $11,45 \%$ com cuidados de alta dependência para o serviço de enfermagem(22). Isso demonstra que as condições também são inadequadas para a equipe de enfermagem, traduzindo-se em sobrecarga de trabalho e diminuição na qualidade da assistência.

Encontramos associação entre a unidade e o tipo de pneumonia, sendo que, no grupo UTI/UTQ/CTQ, a frequência de PAV foi de $34,6 \%$. As UTIs possuem microrganismos de alta patogenicidade que, muitas vezes, colonizam os pacientes, levando a infecções de difícil tratamento. Sabe-se que os fatores de risco se dividem em modificáveis que podem estar associados ao ambiente, e não modificáveis, relacionados ao indivíduo, como idade, escore de gravidade e comorbidades(23).

Além disso, nas UTIs, o atendimento é voltado para usuários com instabilidade sistêmica que possuem maior complexidade nos cuidados e que sofrem com manipulação excessiva e procedimentos invasivos, o que aumenta o risco de infecções(18).

A PAV é uma das complicações mais frequentes em pacientes acometidos por queimaduras, e, frequentemente, encontrada nas UTQs. Muitas vezes, são utilizadas sedações intensas e suporte de VM como tratamento, o que pode estar relacionado com a maior frequência de PAV(24). Ressaltase a implementação de bundles como protocolo institucional que pode reduzir as taxas de PAV, a fim de garantir a segurança 
do paciente(25).

Houve associação significante entre óbito e o tipo de pneumonia, sendo que, dos 146 casos de PAV, 101 evoluíram a óbito. De acordo com Weyland et al.(26), a mortalidade associada à ventilação mecânica varia entre $20 \%$ e $60 \%$, e isso pode ser resultante de vários fatores, como severidade das doenças de base, falência de órgãos e agente etiológico virulento envolvido. Há evidência que o risco de óbito em casos de pneumonia causadas por microrganismos Gramnegativos pode chegar a $68 \%(27)$.

No presente estudo, foi identificado a presença dos tipos de microrganismos por meio da cultura. De acordo com a Associação Paulista de Epidemiologia e Controle de IRAS(28), os agentes etiológicos variam de acordo com o tempo de VM e microbiota de cada instituição de saúde, entretanto, de uma forma geral, são divididos em dois grupos: PAV de início precoce (quando ocorre dentro dos primeiros quatros dias de VM), com predomínio dos agentes com padrão multíssensível: Streptococcus pneumoniae, Haemophilus influenzae, Staphylococcus aureus oxacilina-sensível e as enterobactérias: Escherichia coli, Klebsiella pneumoniae, Enterobacter spp, Proteus spp e
Serratiamarcescens.

$\mathrm{Na}$ PAV de tardio, predomina $\mathrm{O}$ Staphylococcus aureus Oxacilina resistente, bacilos Gram-negativos (BGN) não fermentadores: Pseudomona saeruginosa, Acinetobacter spp e outros BGN com padrão de resistência compatível com betalactamase de espectro extendido (ESBL) e produtores de carbapenemases (gene blaKPC, NDM, etc)(28). De acordo com o estudo "SENTRY Antimicrobial Surveillance Program", os seis principais patógenos responsáveis por $80 \%$ das pneumonias relacionadas à assistência à saúde foram: Staphylococcus aureus (30\%), Pseudomonas aeruginosa (24\%), Klebsiella spp (11\%), Escherichia coli (8\%), Acinetobacter spp e Enterobacter $\operatorname{spp}(7 \%)^{(29)}$.

Diante disso, se justifica a realização de culturas rotineiramente, assim como a realização do teste de sensibilidade aos antimicrobianos. Os principais agentes etiológicos da PAV e a sensibilidade aos antimicrobianos diferenciam entre os serviços, sendo necessária, por isso, uma vigilância epidemiológica na tentativa de contribuir com a instituição de terapêutica adequada. Esses dados mostram a importância do trabalho realizado pela CCIH 


\section{H}

do hospital. Cuidados e estratégias devem ser determinados para cada equipe, respeitando a singularidade local(28).

Com relação à PAV, houve associação com Staphylococcus aureus; são cocos Gram-positivos com características morfológicas e fisiológicas contribuintes para sua virulência. São sensíveis a altas temperaturas, a desinfetantes e soluções antissépticas. Sobrevivem a superfícies secas por um longo período, fazem parte da microbiota de um indivíduo, na epiderme e na nasofaringe em $15 \%$ dos indivíduos sadios. Devido a essas características, é responsável por muitas das IRAS(30).

$$
\text { Como limitação do estudo, }
$$
apontamos a não avaliação da assistência prestada, descrita em prontuários, o que poderia resultar em análise de outras variáveis associadas à pneumonia relacionada à assistência à saúde.

Para a prática e pesquisa, é necessário que ocorram futuros estudos, especialmente estudos longitudinais que acompanhem o paciente com pneumonia desde o diagnóstico até a alta hospitalar ou óbito, além da necessidade de implementação de bundles, garantido, desse modo, que novas normas sejam incorporadas à prática assistencial, reduzindo o risco de eventos adversos, como as IRAS.

\section{CONCLUSÃO}

Houve associação estatisticamente significante entre o tipo e pneumonia e a idade, sendo mais frequente a PAV em jovens. Também houve associação significante entre o tipo de pneumonia e a unidade, sendo mais frequente a PAV nas UTIs, UTQ e CTQ.

A associação entre 0 tipo de pneumonia e óbito também foi estatisticamente significante, sendo que, dos 146 casos de PAV, 101 evoluíram a óbito. A associação com o microrganismo e a pneumonia mais frequente foi a PAV apenas para o Staphylococcus aureus. Entre os dois tipos de pneumonias (PAV e PHA), os microrganismos mais frequentes foram Acinetobacter baumannii, Klebsiella pneumoniae, Staphylococcus aureus e Pseudomonas aeruginosa. 


\section{REFERÊNCIAS}

1. Agência Nacional de Vigilância Sanitária (BR). Medidas de prevenção de infecção relacionada à assistência à saúde [Internet].2 ed. Brasília: Anvisa; 2017 [acesso 2020jun02]. 122 p.Disponível em:http://portal.anvisa.gov.br/document s/33852/3507912/Caderno+4+-

Medidas+de+Preven $\% \mathrm{C} 3 \% \mathrm{~A} 7 \% \mathrm{C} 3 \% \mathrm{~A} 30$ _ +de+Infec\%C3\%A7\%C3\%A3o+Relacion ada $+\%$ C3\%A0+Assist $\%$ C3\%AAncia $+\%$ C 3\%A0+Sa\%C3\%BAde/a3f23dfb-2c544e64-881c-fccf9220c373.

2. Centers for Disease Control and Prevention (US). Ventilator-Associated Event (VAE) For use in adult locations only [Internet].2020 [acesso 2020 jun 06].Disponível em: https://www.cdc.gov/nhsn/pdfs/pscmanu al/10-vae_final.pdf.

3. Agência Nacional de Vigilância Sanitária (BR). Nota Técnica GVIMS/GGTES no 03/2019: critérios diagnósticos das infecções relacionadas à assistência à saúde [Internet]. Brasília: Anvisa; 2019 [acesso 2020 jun 06]. Disponível em: http://portal.anvisa.gov.br/documents/3 3852/271855/Nota+t\%C3\%A9cnica+n\% C2\%BA+03-2019+GVIMS-GGTESANVISA/85f6927c-761d-43bd-ba95b4115bf30600.

4. ManfrediniCS, Marco SL, Denti IA. Prevalência de infecções em uma Unidade de Terapia pediátrica e neonatal. Rev Perspectiva. 2014;38(1):73-81.
5. BakerD, QuinnB, Ewan V, Giuliano Kn K. PhD, RN, FAAN.sustaining quality improvement: long-term reduction of nonventilator hospital-acquired pneumonia. J NursCare Qual. 2019 July/Sept;34(3):223-29. doi: 10.1097 / NCQ.000000000000035

6. Luyt CE, Hékimian G, Koulenti D, Chastre J. Microbial cause of ICU-acquired pneumonia: hospital-acquired pneumonia versus ventilator-associated pneumonia. CurrOpinCritCare. 20180ct;24(5):332-38. doi: 10.1097/MCC.0000000000000526

7. Magill SS, Edwards JR, Bamberg W, Beldavs ZG, Dumyati G, Kaine MA et al. Multistate Point-Prevalence Survey of Health Care-Associated Infections. N Engl J Med. 2014March;370(13):11981208.DOI: 10.1056/NEJMoa1306801

8. Patty C, Sandidge-Renteria A, Orique S, Dixon C, Camarena E, Newsom R, Schneider A. Incidence and predictors of nonventilator hospital-acquired pneumonia in a community hospital. J NursCare. 2020Feb. doi: 10.1097 / NCQ.0000000000000476

9. Silva ALM, Bonfim JV, Santana LS, Guedes LBA. Medidas de prevenção à infecção hospitalar: uma revisão livre na literatura. [Monografia]: Escola Bahiana de Medicina e Saúde Pública: Salvador; 2016. 12p.

10. Souza ES, Belei RA, Carrilho CMDM, Matsuo T, Yamada-Ogatta SF, Andrade G et al. Mortalidade e riscos associados a infecção relacionada à assistência à 
10. saúde. Texto\& Contexto Enfer. 2015jan/mar;24(1):220-28.

http://dx.doi.org/10.1590/010407072015002940013

11. Ladbrook E, Bouchoucha SL, Hutchinson A. Lessons Learned From a Rapid Implementation of a VentilatorAssociated Pneumonia Prevention Bundle. JournalofinfectionPrevention 2019, Vol. 20(6) 274-280. https://doi.org/10.1177/1757177419846 588

12. Lacerna CC, Patey D, Block L, Naik S, Kevorkova Y, Galin J et al. A successful program preventing nonventilator hospital-acquired pneumonia in a large hospital system. Infect Control Hosp Epidemiol. 2020 May;41(5):547-552. doi: 10.1017/ice.2019.368. Epub 2020 Jan 15.

13. Tablan OC, Anderson LJ, Besser R, Bridges C, Hajjeh R. Guidelines for preventing health-care associated pneumonia, 2003: recommendations of $\mathrm{CDC}$ and the Healthcare Infection Control Practices Advisory Committee. MMWR Recomm Rep [Internet]. 2004 march [acesso 2020 jun 06];53(RR03):136. Disponivel em: https://www.cdc.gov/mmwr/preview/mm wrhtml/rr5303a1.htm.

14. Barros MMA, Pereira ED, Cardoso FN, Silva RA. O enfermeiro na prevenção e controle de infecções relacionadas à assistência à saúde. Universitas: Ciências da Saúde [Internet]. Brasília, 2016 jan [acesso 2020 jun 06];14(1):15-21.
14. Disponível https://www.publicacoesacademicas.unic eub.br/cienciasaude/article/view/3411.

15. Baker D, Quinn B. Hospital Acquired Pneumonia Prevention Initiative-2: Incidence of nonventilator hospitalacquired pneumonia in the United States. Am J Infec Control. 2018 Jan;46(1):27.https://doi.org/10.1016/j.ajic.2017.08. 036.

16. Nunes BP, Soares MU, Wachs LS, Volz PM, Saes MO, Duro SMS et al.Hospitalization in older adults: association with multimorbidity, primary health care and private health plan. Rev Saude Publica. 2017 May;51(43):1-10. doi: $10.1590 / \mathrm{S} 1518-$ 8787.2017051006646

17. Ribeiro CL, Barbosa IV, Silva RSM, Cetari VRF,Penaforte KL, Custodio IL. Clinical characterization of patients under mechanical ventilation in an intensive therapy unit. Rev Fund Care Online. 2018 abr/jun;10(2):496-502.

18. Silva TG, Souza GN, Souza SS, Bitencourt JVV, Madureira VF, Luzardo AR. Incidência de pneumonia associada à ventilação mecânica em uma Unidade de Terapia Intensiva. J Rev Fund Care Online. 2017 out/dez;9(4):1121-125. doi: $\quad$ http://dx.doi.org/10.9789/21755361.2017.v9i4. 1121-1125.

19. Mota EC, Oliveira SP, Silveira BRM, Silva PLN, Oliveira AC. Incidência da pneumonia associada à ventilação mecânica em unidade de terapia intensiva. Medicina (Ribeirao Preto 
19. Online) 2017 fev;50(1):39-6. doi:https://doi.org/10.11606/issn.21767262.v50i1p39-46.

20. Di Pasquale M, Aliberti S, Mantero M. Bianchini S. Blasi F. Non-intensive care unit acquired pneumonia: a new clinical entity?Int J Mol Sci. 2016;17(3):287. https://doi.org/10.3390/ijms17030287.

21. Moraes DS, Cordeiro NM, Fonseca ADG, Silva CSO, Souza LPS, Lopes JR. Fatores associados à internação prolongada nas admissões pela urgência e emergência. Rev Univ Vale Rio Verde. 2017;15(2):680-91.

http://dx.doi.org/10.5892/ruvrd.v15i2.37 70

22. Zambonin F, Lima KLB, Brito AR, Brito TB, Amorim RF, Caldart RV. Classification of emergency patients according to their dependency on nursing. J Nurs UFPE online. 2019 Apr;13(4):1133-41. doi: https://doi.org/10.5205/1981-8963v13i04a236792p1133-1141-2019.

23. Costa BHS, Dias TDKC, Paz MSS, Santos CR, Oliveira TC, Marinho HLM et al. Risk factors favoring pneumonia associated with mechanical ventilation. J Nurs UFPE online.2018 Dec;12(12):3401-15. doi: https://doi.org/10.5205/1981-8963v12i12a235025p3401-3415-2018.

24. Gille J, Bauer N, Malcharek MJ, Dragu A, Sablotzki A, Taha $\mathrm{H}$ et al. Reducing the Indication for Ventilatory Support in the Severely Burned Patient: Results of a New Protocol Approach at a Regional Burn Center. J Burn Care Res. 2016 MayJun2;37(3):205-12. doi:
23. $10.1097 /$ BCR. 0000000000000238 .

25. Frota ML, Campanharo CRV, Lopes MCBT, Piacezzi LHV, Okuno MFP, Batista REA. Good practices for preventing ventilatorassociated pneumonia in the emergency department. Rev Esc Enferm USP. 2019;53:e0460. doi: http://dx.doi.org/10.1590/S1980220X2018010803460

26. Weyland B, Perazzi B, García S, Rodríguez C, Vay C, Famiglietti A. Etiología bacteriana de la neumonía nosocomial y resistencia a los antimicrobianos en pacientes con y sin tratamiento antimicrobiano previo. Rev Argent Microbiol. 2011 ene/mar;43(1):18-23. doi: 10.1590/S0325-75412011000100004.

27. Micek ST, Chew B, Hampton N, Kollef MH. A Case-Control Study Assessing the Impact of Nonventilated HospitalAcquired Pneumonia on Patient Outcomes. Chest. 2016 Nov;150(5):1008-14.

28. Associação PaulistaAssociação Paulista de Epidemiologia e Controle de Infecção Relacionada à Assistência à Saúde (BR). Pneumonia associada à assistência à saúde. 3. ed. São Paulo: APECIH; 2019. Capítulo 3, Pneumonia Associada à Assistência à Saúde; p. 35-48.

29. Cilloniz C, Martin-Leoches I, Garcia-Vidal C, San Jose A, Torres A. Microbial Etiology of Pneumonia: Epidemiology, Diagnosis and Resistance Patterns. Int J Mol Sci 2016 Dec;17(12):2120. doi: 10.3390/ijms1712 2120 
30. Moura A Santos; Souza GM; Alves MG; Brito MV; Ladeira LMC; Mendonça VFet al. Resistência bacteriana associada aos casos de infecção hospitalar na Santa Casa de Misericórdia de Passos (MG): estudo de caso. Ciencia Et Praxis [Internet] 2018. [ acesso 2020 Mar 09];11(21):95-106. Disponível em: http://revista.uemg.br/index.php/praxys/ article/download/3886/2278. 